\title{
Studententag informiert über Auslandseinsätze mit Hilfsorganisationen
}

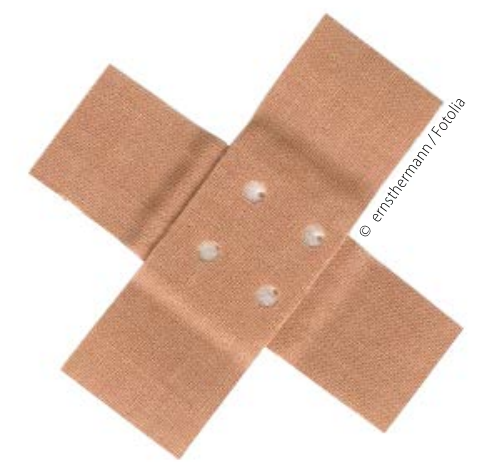

\section{Der diesjährige Studententag beim Deutschen Zahn- ärztetag in Frankfurt am Main spricht auch gestan- dene Zahnmediziner an. Das gilt ebenso für Zahntechniker und Assistenzpersonal. Denn das Pro- gramm unter dem Motto "Famulatur und Auslands- einsatz - Herausforderungen, Möglichkeiten und Realität" bietet reichhaltige Infos über Auslandsein- sätze und zahnmedizinische Hilfsorganisationen.}

Studierende erfahren in der Veranstaltung am 12. November, die der Bundesverband der Zahnmedizinstudenten in Deutschland, die Deutsche Gesellschaft für Zahn-, Mund- und Kieferheilkunde und das Gemeinschaftsprojekt mit dem FVDZ, young dentists $\left(\mathrm{yd}^{2}\right)$, organisieren, wie sie ihre Famulatur mit einem Sinn spendenden Auslandseinsatz verbinden können. Genauso dürfen sich auch junge Zahnärztinnen und Zahnärzte in der Orientierungsphase oder Senioren und Pensionäre angesprochen fühlen.

In Deutschland gibt es verschiedene Organisationen, die im Ausland zahnmedizinisch helfen. Fünf dieser Vereinigungen werden sich in Kurzvorträgen und anschließendem Dialog mit den Teilnehmern vorstellen.

Der Berliner Kammerpräsident, Dr. Wolfgang Schmiedel, gibt zunächst Einblicke in die Grundprinzipien der Entwicklungshilfe und zu den Global Facts. Tiefer steigt Tobias Bauer, Gründer von DIANO (Dental International Aid Networking Organisation), in die Materie ein, der davon überzeugt ist, dass die Übernahme sozialer Verantwortung ein hohes Gut des akademischen Berufsstandes Zahnarzt sei. In seinem Vortrag "Global oral care" wird es um Hintergründe und eine kritische Betrachtung des Themas gehen. Ein Übersichtsvortrag des Zahnmedizinischen Austauschdienstes führt - speziell für Studenten - in die Thematik Famulaturen ein.

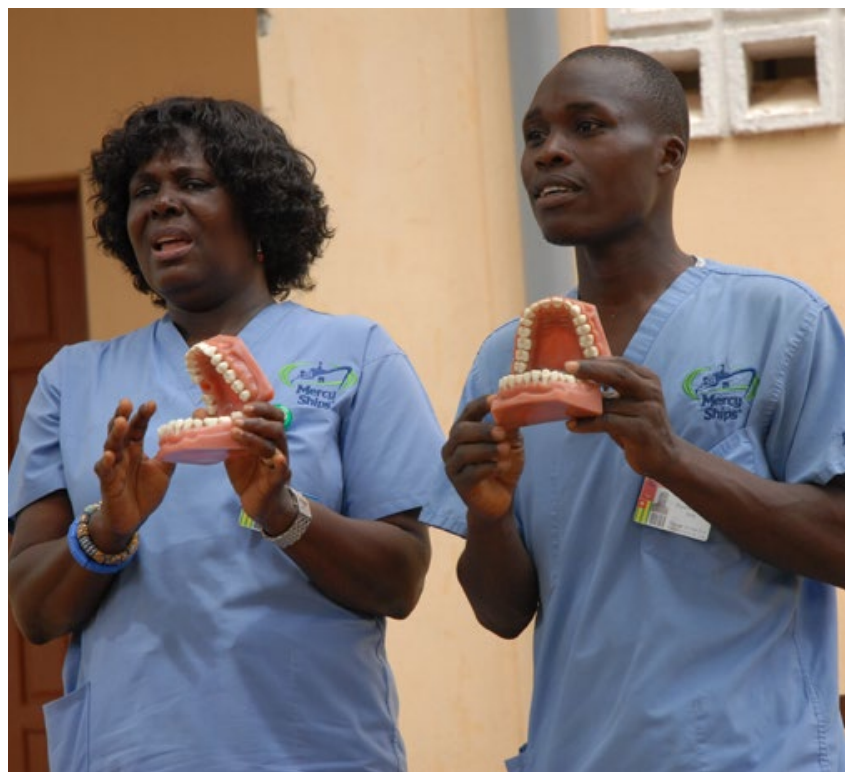

\section{Von Ruanda bis Indien}

Auch die beteiligten Organisationen präsentieren sich. Den Auftakt macht „DENTAL ROOTS - racines dentaires - aktion zahnwurzel e.V.", die eine Graswurzelpartnerschaft mit Ruanda verbindet. In einer Selbsthilfe vor Ort arbeiten Zahnärzte aus Rheinland-Pfalz seit über 30 Jahren mit dem ostafrikanischen Land zusammen, der Verein "DENTAL ROOTS" wurde 2014 gegründet.

Als lose, eng mit der Kindernothilfe (KNH) Duisburg assoziierte Gruppe von Zahnärzten (Zahnärzte für Indien) führt der daraus entstandene Verein GDCI (German Dental Carehood International) seit Mitte der 1980er Jahre dentale Hilfseinsätze in Südindien durch. Zur Zeit werden in Zahnstationen in Missionskrankenhäusern der christlichen „Church of South India“ (CSI) Kinder und Jugendliche aus den unterprivilegiertesten Schichten, die in Heimen und Tagesstätten untergebracht sind, kostenlos zahnärztlich betreut.

Das Zahnärztliche Hilfsprogramm Bayern legt seinen Schwerpunkt auf die Arbeit in Brasilien. Als einmaliges Beispiel für eine internationale Hilfsmission stellen sich die Mercy Ships vor. Darunter die Africa Mercy, das weltgrößte schwimmende zivile Krankenhaus, das international großes Ansehen genießt. Studenten erhalten Reisekostenzuschüsse

Im Anschluss an diese Präsentationen stehen die Vertreter dieser Hilfsorganisationen für Fragen zur Verfügung. Daran schlieBen sich Famulatur- und Projektberichte des BdZM an, in der konkrete Auslandseinsätze auf dieser Basis vorgestellt werden.

Nach einer Zusammenfassung der wesentlichen Informationen für einen Auslandseinsatz wird sich noch die Organisation „Dentists for Africa“ vorstellen.

Studierende können für die Fahrt nach Frankfurt Reisekostenzuschüsse bei der DGZMK beantragen und haben die Möglichkeit, für 15 Euro Registriergebühr den Studententag und das Programm des Deutschen Zahnärztetages zu besuchen. Infos unter www.dtzt.de.

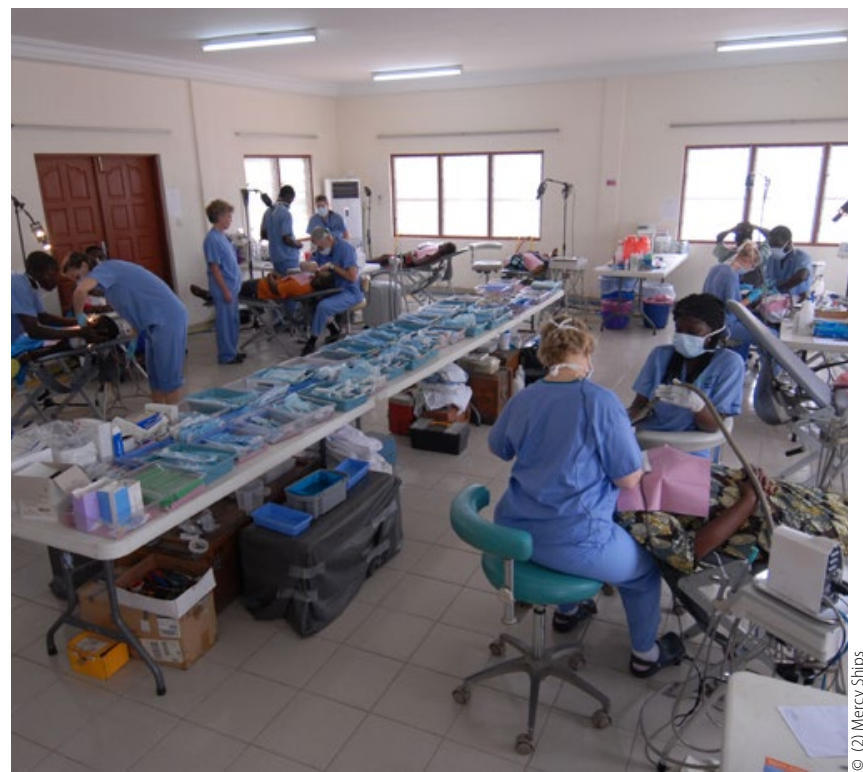

